\title{
From little adults to pediatric patients: quality improvement in pediatric anesthesia
}

\author{
Nicola G. Clausen ${ }^{1,2, \star}$, Tom Giedsing Hansen ${ }^{1,2}$
}

\author{
${ }^{1}$ Department of Anesthesiology and \\ Intensive Care, University Hospital \\ Odense, J.B.Winsloewsvej 4, DK-5000 \\ Odense, Denmark \\ ${ }^{2}$ Department of Clinical Research, \\ University of Southern Denmark, \\ Winsloewparken 19, DK-5000 Odense, \\ Denmark
}

\section{*Correspondence nicola@nicola.dk (Nicola G. Clausen)}

\begin{abstract}
The concept of quality improvement (QI) embraces a systematic approach using specific techniques to improve quality. The objective of this non-systematic review article is to present one concept of QI in healthcare and its application in pediatric anesthesia. As can be shown, QI is well established in pediatric anesthesia. 'Plan-Do-Study-Act' cycles, an approach for improvement in health care in which one learns from taking action, are considered a feasible tool. Quality improvement models do not replace clinical research and scientific publishing, but promote the implementation of new findings into clinical practices.
\end{abstract}

\section{Keywords}

Pediatric anesthesia; Quality improvement

\section{Introduction}

The first fatality due to anesthesia reported in a minor was that of a 15-year old girl scheduled for the removal of an ingrown toenail in 1848. As was the custom in the mid-19th century, the surgeon induced the anesthesia using drops of chloroform poured into a handkerchief, which he held over the mouth and nostrils of the patient [1]. The girl died shortly after, although attempts were made to resuscitate her from what appeared to have been convulsions and pulmonary edema [2]. At that time and for many decades to follow, the clinical anesthesia practices in children were based on experiences from adults and assumptions made about the pediatric physiology and pharmacology rather than on carefully conducted research. In fact, pediatric anesthesia as a subspecialty cannot be dated back any further than to the first third of the 20th century, when pioneers like Charles H. Robson described the digital tracheal intubation in children [3] and Dr. Philip Ayre developed the pediatric breathing circuit [4]. In modified forms, Ayre's Tpiece is still in clinical use today.

Throughout the 20th century, distinct features related to anesthesia and care of children were increasingly acknowledged, e.g., emotional challenges correlated to the child's stage of cognitive development, anatomical and pharmacological differences. In 1975, Smith and Steward set up the '5 Goals of Pediatric Anesthesia' [5]. In summary, Smith summoned pediatric anesthesiologists to support the progressing pediatric surgical expertise through increased anesthetic skills and to develop the specialty through research and education. The overall aim was to reduce anesthesia-related morbidity and mortality and to improve resuscitation and supportive care of children. Although there is no clear definition of 'quality in pediatric anesthesia', one would agree, that clinical practices based on scientific evidence, improved patient outcome and standards for educational curricula, as Smith asks for, correspond well to an understanding of 'quality'. However, Smith did not describe a framework or manual, as how to achieve these goals.

The concept of quality improvement (QI) embraces a systematic approach using specific techniques to improve quality. The objective of this non-systematic review article is to present one concept of QI in healthcare and its application in pediatric anesthesia.

\section{The model of quality improvement in healthcare}

Originally, the concept of QI was invented in the production industry to reduce cost and eliminate defects. A line of production was identified as a system: a set of interdependent elements interacting to achieve a common purpose. Berwick and Nolan translated this view into the clinical setting and argued, that healthcare, too, is a process rather than the sum of individual actions $[6,7]$. They realized that the change of one minor element in the process could have a large impact on the product [8]. Hence, systemic improvement required cumulative and linked changes in an ongoing series of tests or 'circles of change'. This attitude has since allowed healthcare professionals to focus on the system rather than the individual.

The most commonly used approach for cycle improvement in health care is the use of Plan-Do-Study-Act (PDSA) cycles in which one learns from taking action [9]. This method involves a "trial-and-learning" approach: a hypothesis or suggested solution for improvement is tested, following a sequence of four repetitive steps. In the first plan $(\mathrm{P})$ phase, ideas for improvement are detailed, tasks assigned, and expectations 
confirmed with the testing team. Measures of improvement are selected as part of the $\mathrm{P}$ phase. In the $d o$ (D) phase, the plan is implemented, and any deviations from the plan, i.e., defects, are documented. The defects are analyzed in the study (S) phase. In this phase the whole course of the cycle is evaluated. In the act (A) phase, lessons learned from the $\mathrm{S}$ phase are incorporated into the test of change, and a decision is made about continuation of the test cycles. For the next cycle, the four steps are repeated. The duration of each cycle depends on the subject and complexity of the project.

Quality improvement efforts focus primarily on making care better at local sites, rather than on generating new, generalizable scientific knowledge. Despite its local focus, improvement frequently generates important knowledge about systems of care and about how best to change those systems. Hence, the reporting and propagation of QI results are of value. Unfortunately, relatively little of that work is reported in the biomedical literature, and much of what is published could be more effectively presented. In an effort to strengthen the evidence base for improvement in health care, the Standards for Quality Improvement Reporting Excellence (SQUIRE) guidelines were introduced. The SQUIRE guidelines "provide a framework for reporting new knowledge about how to improve healthcare" (wWw. squire-statement.org).

Similar to scientific research, QI projects involve defining a problem and drafting a potential solution to solve it, which is implemented clinically. The effect is then monitored and in some cases compared to standard treatment or placebo. It should be emphasized, however, that QI is no substitute for scientific research, although overlaps between these two approaches exist [10]. The two approaches differ substantially with respect to several aspects. In QI, a known solution is applied in order to achieve whatever objective was set: cost reduction, accelerated patient flow, quality, just to name a few. In comparison, the aim of research, is to generate new knowledge. Whereas QI is most often conducted locally and the results not generalizable, research can be conducted as uni- or multicenter studies and, if properly designed, the study results should be valid and reproducible elsewhere. For the patients involved, QI projects should not pose any increased risk and hence informed consent and approval by official agencies and institutional research boards is not required.

In practice, even though they originated in studies with high internal and external validity, study results are often difficult to reproduce in different locations. This does not mean that the association between an intervention and an outcome is untrue. But it demonstrates, that changes in healthcare require more than specialty specific knowledge. In addition, profound knowledge is needed, comprising an understanding of the system to be changed and, most importantly, how to facilitate this change [11]. In summary, QI models can support the implementation of 'best practices' and standards, once these have been established based on evidence from scientific research. Its objective is to improve outcomes in healthcare by improving healthcare processes, rather than improving the outcomes alone.

\section{Quality improvement in pediatric anesthesia}

In 2013, the Pediatric Anesthesia journal launched a themed issue dedicated to QI. Besides explaining the method of quality improvement, the issue contains multiple original articles on the subject, which exemplify application of the QI model in diverse areas of pediatric patient care. Since then, a multitude of pediatric anesthesia QI projects have been published in 'Pediatric Anesthesia' as well as in other journals. Some have addressed a well-defined problem, e.g., blood product utilization in pediatric cardiac surgery after introduction of a blood product transfusion algorithm [12] or a reduction in the rate of unplanned extubations in a pediatric intensive care unit [13], to name a few examples. Projects in a larger scale comprise 'Wake Up Safe' and 'APRICOT'. The US Society for Pediatric Anesthesia sponsors 'Wake Up Safe', a registry of defined serious adverse events occurring during the perioperative period [14]. Contributing institutions - currently 29 centers in Northern America-report data on patient demographics and adverse events, including a structured analysis of why these events occurred as well as their preventability [15]. The registry was established in order to identify areas in patient care in need of improvement [11]. It is based on specific components: (1) 'smart aims' defined in advance as the goal(s) for the program; (2) 'key drivers', that is factors required to support the program (e.g., financial and human resources, knowledge dissemination and institutional leadership support for implementation of QI initiatives) and lastly (3) interventions performed to make the key drivers fully operational. Since its development almost a decade ago, the Wake Up Safe Collaboration has contributed with reports on multiple relations of high importance for the quality in pediatric anesthesia [16-19]. The latest being a report on perioperative anaphylaxis in children published early in 2021 [19]. Overall, the analysis of reason for and preventability of adverse events is considered a great strength of the Collaboration.

Another initiative, the European APRICOT Study, reported data on perioperative adverse events in pediatric anesthesia cases collected in 261 participating centers across 33 European countries [20]. Due to inclusion of international data, APRICOT represents an accurate snapshot of events occurring in European daily clinical practice in pediatric anesthesia. In comparison to the Wake Up Safe Collaboration, however, an analysis of why adverse events occurred was not part of the APRICOT study protocol. It is hence up to each participating institution and country to recap and interpret the course of actions leading to an adverse event. Since the initial publication of the APRICOT Study report, a range of secondary analysis of specific types of adverse events or national circumstances have been conducted [21-27]. However, based on data from the APRICOT Study alone, adverse events cannot be prevented. Data collection was restricted to assessment of quantitative outcomes rather than on the course of consecutive actions and happenings resulting in a given event. Merely identifying and quantifying adverse events does not prevent them from occurring. Neither does it improve quality of care. But studies like APRICOT provide pediatric anesthetists with 'baseline data', defining potential issues which require improvement. They are 
hence an important prerequisite for asking relevant questions and designing appropriate and feasible QI processes, as well as being a platform for political pressure for organizational and educational changes.

\section{Conclusions}

The combination of a well-described systematic approach and reporting standard has made QI an endorsed method commonly used to improve the care of children. With the ever-increasing focus on quality of care inside the hospital system, these QI methods are likely to be used a lot more in the future. In this context, PDSA cycles are considered a feasible tool. Quality improvement models do not replace clinical research and scientific publishing, but promote the implementation of evidence based findings into clinical practices.

\section{AUTHOR CONTRIBUTIONS}

NGC and TGH conceived the original idea; NGC reviewed the literature and TGH contributed to the interpretation of results; NGC wrote the manuscript with inputs from TGH.

\section{ETHICS APPROVAL AND CONSENT TO PARTICIPATE}

Not applicable.

\section{ACKNOWLEDGMENT}

Not applicable.

\section{FUNDING}

This research received no external funding.

\section{CONFLICT OF INTEREST}

The authors declare no conflict of interest.

\section{REFERENCES}

[1] Mai CL, Coté CJ. A history of pediatric anesthesia: a tale of pioneers and equipment. Pediatric Anesthesia. 2012; 22: 511-520.

[2] Lyman HM. Artificial Anaesthesia and Anaesthetics. New York: William Wood and Company. 1881.

[3] Robson CH. Anesthesia in children. Anesthesia \& Analgesia. 1925; 4: 235-240.

[4] Ayre P. Anaesthesia for hare-lip and cleft palate operations on babies. British Journal of Surgery. 1937; 131-132.

[5] Smith R. The pediatric anesthetist, 1950-1975. Anesthesiology. 1975; 43: 144-155.

[6] Varkey P, Reller MK, Resar RK. Basics of quality improvement in health care. Mayo Clinic Proceedings. 2007; 82: 735-739.

[7] Berwick DM. Continuous improvement as an ideal in health care. New England Journal of Medicine. 1989; 320: 53-56.

${ }^{[8]}$ Berwick DM, Nolan TW. Overview: cooperating for improvement. The Joint Commission Journal on Quality Improvement. 1995; 21: 573-577.

[9] Berwick DM. Developing and testing changes in delivery of care. Annals of Internal Medicine. 1998; 128: 651-656.
[10] Gregory KE. Differentiating between research and quality improvement. The Journal of Perinatal \& Neonatal Nursing. 2015; 29: 100-102.

[11] Kurth CD. Introducing quality improvement. Paediatric Anaesthesia. 2013; 23: 569-570.

[12] Whitney G, Daves S, Hughes A, Watkins S, Woods M, Kreger M, et al. Implementation of a transfusion algorithm to reduce blood product utilization in pediatric cardiac surgery. Paediatric Anaesthesia. 2013; 23: 639-646.

[13] Rachman BR, Mink RB. A prospective observational quality improvement study of the sustained effects of a program to reduce unplanned extubations in a pediatric intensive care unit. Paediatric Anaesthesia. 2013; 23: 614-620.

[14] Kurth CD, Tyler D, Heitmiller E, Tosone SR, Martin L, Deshpande JK. National pediatric anesthesia safety quality improvement program in the United States. Anesthesia and Analgesia. 2014; 119: 112-121.

[15] Haché M, Sun LS, Gadi G, Busse J, Lee AC, Lorinc A, et al. Outcomes from wake up safe, the pediatric anesthesia quality improvement initiative. Pediatric Anesthesia. 2020; 30: 1348-1354.

[16] Christensen RE, Lee AC, Gowen MS, Rettiganti MR, Deshpande JK, Morray JP. Pediatric perioperative cardiac arrest, death in the off hours: a report from wake up safe, the pediatric quality improvement initiative. Anesthesia and Analgesia. 2018; 127: 472-477.

[17] Christensen RE, Nause-Osthoff RC, Waldman JC, Spratt DE, Hearn JWD. Adverse events in radiation oncology: a case series from wake up safe, the pediatric anesthesia quality improvement initiative. Paediatric Anaesthesia. 2019; 29: 265-270.

${ }^{[18]}$ Lobaugh LMY, Martin LD, Schleelein LE, Tyler DC, Litman RS. Medication errors in pediatric anesthesia: a report from the wake up safe quality improvement initiative. Anesthesia and Analgesia. 2017; 125: 936-942.

[19] Wakimoto M, Miller R, Kim SS, Uffman JC, NafiuS OO, Tobias JD, et al. Perioperative anaphylaxis in children: a report from the Wake-Up Safe collaborative. Pediatric Anesthesia. 2021; 31: 205-212.

[20] Habre W, Disma N, Virag K, Becke K, Hansen TG, Jöhr M, et al. Incidence of severe critical events in paediatric anaesthesia (APRICOT): a prospective multicentre observational study in 261 hospitals in Europe. The Lancet Respiratory Medicine. 2017; 5: 412-425.

[21] Dadure C, Veyckemans F, Bringuier S, Habre W. Epidemiology of regional anesthesia in children: lessons learned from the European Multi-Institutional Study APRICOT. Pediatric Anesthesia. 2019; 29: $1128-1135$.

[22] Engelhardt T, Ayansina D, Bell GT, Oshan V, Rutherford JS, Morton NS. Incidence of severe critical events in paediatric anaesthesia in the United Kingdom: secondary analysis of the anaesthesia practice in children observational trial (APRICOT study). Anaesthesia. 2019; 74: 300-311.

[23] Engelhardt T, Virag K, Veyckemans F, Habre W. Airway management in paediatric anaesthesia in Europe-insights from APRICOT (Anaesthesia Practice in Children Observational Trial): a prospective multicentre observational study in 261 hospitals in Europe. British Journal of Anaesthesia. 2018; 121: 66-75.

[24] Habre W. Pediatric anesthesia after APRICOT (Anaesthesia PRactice in Children Observational Trial): who should do it? Current Opinion in Anaesthesiology. 2018; 31: 292-296.

[25] Hansen TG, Børke WB, Isohanni MH, Castellheim A. Incidence of severe critical events in paediatric anaesthesia in Scandinavia: secondary analysis of Anaesthesia PRactice in Children Observational Trial (APRICOT). Acta Anaesthesiologica Scandinavica. 2019; 63: 601-609.

[26] Michel F, Constant I. Critical events in paediatric anaesthesia: lessons learned from the APRICOT study's French data. Anaesthesia Critical Care \& Pain Medicine. 2019; 38: 599-600.

[27] Virag K, Sabourdin N, Thomas M, Veyckemans F, Habre W. Epidemiology and incidence of severe respiratory critical events in ear, nose and throat surgery in children in Europe: a prospective multicentre observational study. European Journal of Anaesthesiology. 2019; 36: 185-193.

How to cite this article: Nicola G. Clausen, Tom Giedsing Hansen. From little adults to pediatric patients: quality improvement in pediatric anesthesia. Signa Vitae. 2021;17(4):1-3. doi: $10.22514 / \mathrm{sv} .2021 .088$. 